\title{
NAVEGAR Y REZAR \\ DEVOCIÓN Y PIEDAD DE LAS GENTES DE MAR BARCELONESAS \\ (SIGLOS XIV Y XV)
}

\author{
$M^{a}$ ELISA VARELA \\ Universitat de Girona
}

La gente de mar' constituye un grupo original y bastante cerrado. Estas gentes conservan sus peculiaridades ${ }^{2}$ con independencia de los espacios regionales de los que son originarios y a lo largo de amplios períodos cronológicos.

Los marinos tenían una visión del mundo con un gran número de elementos comunes como grupo y cada uno conocía las características de la contratación, de los salarios, de la disciplina o de la alimentación que regían entre sus colegas.

Estas características y constantes comunes no sorprenden tanto si pensamos que son personas que permanecían mucho tiempo fuera de sus hogares, meses y algunos incluso años, y este alejamiento les confería, sin duda, una percepción diferente de muchos aspectos vitales: de los vínculos familiares, vecinales e incluso de algunas prácticas o devociones.

\footnotetext{
'Denominación que incluye, como dice G. Airaldi, a todo aquel que opera en el mar, con independencia de la función que realice. Vid. G. AIRALDI, Marineros, etnias y sociedades en el Mediterráneo medieval. El caso de Génovia, en El mundo mediterráneo de la Edad Media, Barcelona, 1987, p. 166. Es decir, en la expresión "gentes de mar" encuadramos a todos los componentes de las tripulaciones - a los que llamaremos "marinos", tomando el término en su acepción más amplia-. a los mercaderes que realizaban sus operaciones por mar, a los barqueros, pescadores y a todos los oficios vinculados con el trabajo en el mar, con sus esposas y familias.

'El primer elemento que les confiere singularidad a las personas del mar es su lenguaje. Aunque los marinos comparten la lengua de las gentes del territorio del que son originarios, utilizan un lenguaje profesional que traspasa el ámbito de su profesión y afecta a la vida ordinaria. El lenguaje marinero está plagado de términos específicos que resultan incomprensibles, muchas veces, para las gentes que no viven del mar. Un lenguaje que los diferencia de otros grupos y que obedece y da cuenta de una realidad vital y profesional profundamente distinta de la terrestre.
}

"Anuario de Estudios Medievales". 29 (1999) 
Elementos destacables son, también, la continuidad de las familias que se dedicaban al trabajo en el mar, la solidaridad nacional y la gran diversificación de la actividad de los grupos familiares ${ }^{3}$, aunque algunas familias se especializan en alguna labor determinada.

Una constante destacable del ámbito catalán y mediterráneo es la gran movilidad ${ }^{4}$, la circulación constante de todos aquellos que se dedican a la navegación y a trabajar en y con las naves, frente a un alto grado de estabilidad de las familias que se dedican a otros oficios de mar. En los puertos catalanes y, en especial, en Barcelona se encuentran marinos de casi todo el Mediterráneo y de otros ámbitos como los vizcaínos, cántabros, gallegos, etc.

El estudio de la devoción de las gentes de mar permite observar que los elementos más característicos no pertenecen al campo o al dominio de la piedad. En éste ámbito forman un grupo permeable a las grandes modas y a las particularidades y costumbres de su zona geográfica de procedencia ${ }^{5}$.

Es necesario también subrayar que la gente de mar era, como cualquier otro estrato social bajo-medieval, un grupo bien estratificado socialmente. El colectivo más marginal estaba representado por la marinería, que era considerada el colectivo más "distinto, el más diferente" dentro del mundo del mar y era el más dependiente del medio marítimo ${ }^{6}$.

El primero o uno de los primeros elementos que refleja el sentido religioso y las devociones de los hombres de mar es el nombre de las embarcaciones $^{7}$ en las que trabajan. En Cataluña, durante el siglo XIII y las primeras décadas del siglo XIV, aún pervivían en las embarcaciones nombres de animales cuyo principal atributo era la velocidad (Gatzela, Gavina, Llamp, etc.) o nombres cariñosos (Oliveta, Monaguina, etc. $)^{8}$. Estos

\footnotetext{
${ }^{3}$ Se puede mencionar la pesca, el transporte, el comercio, la guerra, el corso, etc.

${ }^{4}$ T.-M. VINYOLES I VIDAL, La vita quotidiana della gente di mare (Esempi barcellonesi dei secoli XIV e XV), en "Medioevo. Saggi e Rassegne", 21 (1997), pp.9-35.

${ }^{5}$ Vid. H. BRESC, La pieté des gens de mer en Mediterranée occidental anx derniers siècles du Moyen-Agge, en Le Genti del Mare Mediterraneo a cura de Rosalba Ragosa, Nápoles. 1981. p. 428.

'J.E. RUIZ DOMÉNEC, El sueño de Ulises: la actividad marítima en la cultura mediterránea como un fenómeno de estructura, en Le genti del mare Mediterraneo, op. cit., p. 33.

${ }^{7}$ Vid. N. ColL, Noms de galeres catalanes del segle XV en "Butlletí de la Societat d'Onomàstica", XXXII (juny, 1988), pp. 35-40 y, A. GarCía SANZ i N. Coll, Galeres mercants catalanes dels segles XIV i XV, Barcelona, 1994, pp. 37-54 y 202.

${ }^{8}$ Vid. A. García Sanz-N. Coll, op. cit., p. 202.
} 
nombres, ya desde el siglo XIII, coexisten con nombres que invocan la protección celestial, costumbre que se acentúa a lo largo del siglo XIV y se generaliza en el siglo XV.

A. García y N. Coll sostienen que los nombres de santos dados a las galeras catalanas, de los siglos XIV y XV, son los mismos de las invocaciones de las Letanías ${ }^{9}$ recitadas a bordo, tanto en el acto previo a la salida de puerto, la salutació ${ }^{10}$, como en otros momentos de la singladura. Los más frecuentes son: "Santa Maria, Sant Miquel, Sant Gabriel, Sant Joan, Sant Pau, Sant Pere, Sant Antoni, Sant Nicolau, Sant Francesc, Santa Caterina, Santa Àgata", etc.

Los testamentos ${ }^{11}$ son una de las fuentes que muestran más directamente las devociones y la piedad de las gentes de mar, de sus esposas y de aquellas personas con las que se asociaban en el medio portuario (mercaderes, calafates, barqueros, etc.). Estos mismos aspectos pueden apreciarse, también, en algunos libros de contabilidad privada de mercaderes. Estas últimas fuentes y, sobre todo, las cuentas de los ejecutores testamentarios ofrecen, todavía mejor que los testamentos, las formas concretas a que daban lugar la devoción y la piedad de la gente de mar ${ }^{12}$. Testamentos y libros de contabilidad son, básicamente, los dos tipos de fuentes que utilizaremos en este trabajo.

Los libros de contabilidad ${ }^{13}$ de los mercaderes que analizamos recogen manifestaciones diversas de piedad y devoción. Aportan alguna

\footnotetext{
9Tal como señalan García Sanz y Coll, "les Lletanies dels Sants", son -en el siglo XIVuna plegaria colectiva. Vid. op. cit. p. 203 y nota 156.

${ }^{10}$ Vid. A. García Sanz-N. Coll, op. cit., p. 203.

"Agradezco a la Dra. Pinuccia Simbula, de la Università di Sassari, que me haya permitido consultar los testamentos de gentes de mar que había recogido y fichado en l'Arxiu Històric de Protocols de Barcelona.

${ }^{12}$ Los libros de cuentas recogen diversos aspectos de la organización, no sólo del comercio marítimo, sino también, de la vida a bordo en las distintas singladuras (listas de contratación de los tripulantes, salarios, el trabajo realizado a bordo, la duración del contrato, las cantidades avanzadas a cuenta, utensilios e instrumentos, ropas, armas, comida, etc.).

${ }^{13}$ Analizamos los libros de contabilidad privada de Bernat y Jaume Tarascó. Datados entre 1329 y 1348. Los tres libros están depositados en el Archivo de la Catedral de Barcelona (ACB) en el fondo "Llegats de Llibres Extravagants". Sección "Comptes de companyies, comerciants, navegants, individus, etc.". "Llibre de comptes de Bernat Tarascó (1329-1336)" que citaremos como (Libro I). "Llibre de comptes de Jaume Tarascó (1334-1338)" al que nos referiremos como (Libro II) y "Llibre de comptes de Jaume Tarascó (1340-1348)" que será citado como (Libro III).
} 
oración ${ }^{14}$, diversas fórmulas en las que se encomiendan y se ponen bajo la protección celestial ${ }^{15}$, algunas ofrendas ${ }^{16}$ y un gran número de invocaciones ${ }^{17}$.

${ }^{14} \mathrm{La}$ plegaria da cuenta de la divulgación popular de alguna oración probablemente introducida a partir de san Bernardo. Es, además, una bella muestra de la proximidad entre el registro oral y el escrito. "Jesuchrist fill de Déu viu, qui per la volentat del Pare e per obre del Sant Erspirit et per la tua sancta misericòrdia vengits en aquest món e prengist carn umana, e nasquist del cors de la gloriosa Verga Madona Santa Maria, e donest babtisme e penetènsia e sacrifisi, ab sagrament de Santa Esgléya, en remesió de pecats, e soferits per nós fam e set e calor e fret e engoxes e tribulacions et nalananses, e fuyt pesaguit e venut e pres et ligat e asotat, enalmenat et farit, en la care escopit, el cap d'espines coronat, et de fell et de vinagra abeurat, et mor et lansayat e per la nostra redempció, Senyor meu. devalest-te'n en infern e treguist-ne los teus amichs e puyest-los al sell en la tua alta vertut gloriosament, e ceus a la part dreta dell teu Pare, et vendras al jorn del judici per jutyar los vius e-lls morts e deràs a cascun son guardó, segons que aurà sarvit. Tú est, Déu, Senyor meu, creador, redemptor, refugi, glòria, amor, dousor, part, esperansa mia, Tú, Senyor meu creueador. E lou e benehesch et a Tú, Sanyor, fas lahors et gràcies del bé et de la onor et de la gràcia e de la misericòrdia que as feta a mi, pecador, et a tota crestientat. A Tú, Senyor meu, me confés e pene't-me dells malls que he feyts, molt de mall e fet, de què-n pogrà éser estat molt, deben pegrà aver fet que no he fet de tot ne pinetància, Sanyor meu, et dich mia colpa. E plàsia a Tú, Sanyor meu, qui perdonest a madona santa Maria Machdelena et a mon sanyor sant Pere et a mon sanyor sen Poll e al ladre en la creu, et reps a mersè tots los penedens, que Tú, Sanyor, ayes [mersè] de $\mathrm{mi}$, pecador, e perdone $\cdot \mathrm{m}$, Sanyor, sagons la tua gran pietat et sagons la tua santa misaricòrdia, et mostre- $m$ a fer et a dir la tua santa volentat et, a tanir del dia de la mia vida entrò al dia de la mia fi, en totes lers mies cuyters. Sanyor, te clam verament mersè que $\cdot m$ síhes ajudedor, governedor, defanador et empare $\mathrm{m}$ la mia ànima pecadora $\mathrm{e} \cdot \mathrm{l}$ meu ersperit en les tues santes, beneytes mans, et done-m vida perduratble al teu preciós regna per omnia secula seculorum, ament. Déus sia ab nós et ab tots pecadors". Vid.. Libro I, fol. 98v.

${ }^{15}$ Fórmulas como: "... Déus per sa pietat nos lexs fer bona fí a nós et a tots pecadós ...". Vid. Libro I, fol. 59. "... Déus los lexs ben fer et ben dir et ells don salvament ...". Vid. Ibidem. "... Déus los lexs anar et tornar sansés et saüll, ament ....". Vid. Libro I, fol. 64v. ".. Déus li don salvament et guany a hell et a tots sells qui a bon entaniment van ...". Vid. Libro I, fol. 83v. "Pesà de questa vida en Bernat, fill meu,... Déus li perdó sos pecats". Vid. Libro I, fol. 97v. col. b. "... Ab lo nom de Nostre Sanyhor Déus Jesuchrist ... nasch ... lo yorn de Madona Santa Maria Machdelena, en Jacme, fill nostro ... Deus per sa mersè li don gràcia que sia bo et prom et l'aport Nostre Sanyor a bona fi, ament ...". Vid. Libro I, fol. $98 \mathrm{col}$. b. ".. Pasà de sta vida ... ... Déus los perdó, ament". Vid. Libro I, fol. 104. "... hi guanats so que Déus vulla ... e n'ayats al menys caball e guany, Déus hi derà de la sua grasia". Vid. Libro II, fol. $17 \mathrm{v}$. "... Déus li do molt da bé ... ...... Déus per la sua mercè li perdó sos pechats ament". Vid. Libro III, fol. Iv. "... quants Déu vulla que siam en Pisa ... salvament que Deus hi do ...". Vid. Libro III, fol. 13v. col. a.

16 "Ítem Ia liura de canelles ...". Libro II, fol. 34. "Ítem per Sancta Maria ... I s.". Vid. Libro III, fol. $143 \mathrm{col}$. b.

17"Ab lo nom de Déu ...". Vid. Llibre de comptes de Bernat Tarascó (Libro I), fol. 46v "Ab lo nom de Déu et de Madona Santa Maria ...". Vid. Libro I, fol. 49. "Ab lo nom de Nostre Sanyhor et de Madona Santa Maria ...". Vid. Libro I, fol. 55v. col. a. "... Tot quan fasam sia ab lo nom de Nostre Sanyor Deus Jesuchrist et de Madona Santa Maria ...". Vid. Libro I, fol. $95 \mathrm{col}$. a. "... Ab 10 nom de Nostro Sanyorh Deus Juesuchrist et de la Verga Madona Santa Maria. Nasch en Bernat, fill meu ...". Vid. Libro I, fol. 97v. col. a. "[En nom de Nostre] Senyor Christ. Amen ...". Vid. Libro II, fol. 1. "Ab lo nom de Déu primerament ...". Vid. Libro II, fol. 2. "In nomine Domini, ament. Ab lo nom de Nostre Senyor partim ...". 
Las invocaciones utilizadas en estas fuentes recogen la devoción a Cristo, al Cristo Salvador, que se extiende por diversas áreas mediterráneas ${ }^{18}$. Muestran la menor frecuencia de la invocación con el simple nombre de Dios y permiten constatar que la invocación a Jesús y a la Trinidad son devociones más tardías, más habituales en el siglo XV. En ninguno de los libros catalanes estudiados se recoge la invocación al Espíritu Santo ${ }^{19}$.

Al igual que las restantes prácticas devocionales, también la invocación se extiende por todo el ámbito mediterráneo y se amolda a los deseos del clero: la Virgen jugará - desde el siglo XIII- ${ }^{20}$ un papel fundamental en la piedad de los marinos.

Se puede apreciar que en situaciones de peligro, durante algún temporal que ponía la embarcación al borde del naufragio, algunos miembros de la tripulación realizaban promesas y rogativas especiales -ofrendas de velas, misas, etc. - .

Se intuye, con claridad, que una vez pasado el peligro, en ocasiones, los tripulantes se olvidaban de la promesa o rogativa ofrecida y, por tanto, no la cumplían. Estas ofrendas o peticiones para propiciar la protección divina vuelven a aparecer en los testamentos para que los albaceas las ejecuten después de la muerte del oferente.

También es una tarea de los albaceas organizar otras prácticas rituales y piadosas, entre ellas, la comida para los parientes y amigos el día del entierro ${ }^{21}$. Esta práctica, también aparece documentada entre las gentes de mar. Citaremos a modo de ejemplo el entierro del patrono de nave,

Vid. Libro II, fol. 7. "... En nom de Nostro Ssenyor Deus Jesuchrist ssia ...". Vid. Libro II, fol. 67. "En nom de Nostro Sanyor Deuss Jesuchrist, assò éss libre ...". Vid. Libro II, fo. 40. "Jesus. En nom de Nostre Sanyor Deus Jesucrist". Vid. Libro III, fol. 1.

${ }^{18} \mathrm{H}$. Bresc menciona la Liguria, la Toscana y, también el área catalana y siciliana donde se mantendrá largo tiempo. Cfr. H. BRESC, op. cit., p. 433.

${ }^{19} \mathrm{La}$ invocación al Espíritu Santo es, según $\mathrm{H}$. Bresc, característica de las tierras ligures y sicilianas. Vid. H. BRESC, op. cit., p. 433.

${ }^{20}$ En Barcelona esta devoción a la madre de Cristo se extenderá con mayor rapidez que en las restantes tierras catalanas.

${ }^{21}$ El testador dejaba una cantidad de dinero de la herencia para invitar a una comida a aquellos familiares y amigos que fuesen a su entierro. Es una comida que tiene como fin recordar al difunto y agradecer a aquellos que lo acompañan que lo recuerden el día de su entierro, por tanto se puede calificar como una colación ritual. 
Salvador Orta $^{22}$ y el de la mujer de un barquero que también cumple con el rito de la comida funeraria ${ }^{23}$.

La mayoría de barceloneses escogían como lugar de enterramiento el cementerio de su parroquia, pero, también había gente que prefería los de los diversos monasterios -sobre todo Fra Menors ${ }^{24}$ y el de la catedral ${ }^{25}$.

Los testamentos no permiten deducir, con claridad, cuál era el criterio para la elección de cementerio, podía obedecer a una devoción, o a la existencia de una tumba en propiedad por parte de la familia ${ }^{26}$ o de la cofradía $^{27}$, y en la que ya reposa algún ser querido.

La preferencia por el cementerio parroquial parece lógica si tenemos en cuenta que es la parroquia la que organiza y dirige el culto. Los habitantes de los diferentes barrios de la ciudad estaban adscritos a la parroquia que les pertenecía por zona, y cuando necesitaban cualquier

\footnotetext{
${ }^{22}$ En el que las gentes que estaban presentes -con seguridad un buen número de marinos y gentes del puerto- debieron comer y beber con abundancia: se gastaron dieciocho kilos de carne de carnero, dos docenas de huevos y salsa abundante y no se habría escatimado ni el pan ni el vino. A lo largo de la comida el ambiente se relajaría y dejaría cada vez más espacio a las bromas y a la fiesta. Parecen dar cuenta de ello las sumas que se añaden para pagar la gran cantidad de tazas de vidrio que se rompen y que se alquilaban, al igual que otros utensilios, para poder atender a los invitados. AHPB (Arxiu Historic de Protocols de Barcelona), Bernat Nadal, "Secundus Liber Diversorum Manumissoriorum", fol. 140-140v.

${ }^{23} \mathrm{El}$ año 1402 , los albaceas de la esposa de un barquero que vivía en el barrio de Ribera se ven obligados, dadas las reducidas dimensiones de la casa, a alquilar "scanorum que portam fuerunt ante portale hospicii, in quibus sederunt parentes et amici qui interfuerunt sepulture" y también alquilaron las tazas y escudellas. Los invitados podrán sentarse para comer el pan, vino, espinacas y pescado - porque era Cuaresma - que fue lo ofrecido en esta comida de funeral. AHPB, Bernat Nadal, "Secundus Liber Diversorun Manumissoriorum", fol. 83v.

${ }^{24}$ Este es el caso de Valença, esposa del marinero Guillem Leopardi, que en su testamento datado el 27 de diciembre de 1375, pide ser enterrada en el cementerio de la iglesia de la orden franciscana y, además, quiere ser sepultada con el hábito de la orden y, señala que su parroquia era Sant Just. Ramon de Massana, "Capibrevium testamentorum et codicillorum", 1371-1376, fol. $52 \mathrm{v}$.

${ }^{25} \mathrm{La}$ elección del cementerio de la catedral de la ciudad parece estar poco arraigada entre las gentes de mar, ninguno de los testamentos consultados menciona esta elección para la sepultura.

${ }^{26}$ Este es el caso del marinero Martín de Segura que quiere ser enterrado en el cementerio de Santa María del Mar "in tumulo ubi requiescit Boneta filiam meam". Testamento otorgado en Barcelona el 20 de abril de 1493. Guillem Jordà, "Tricesimum quartum manuale", 2 gener 1493-23 juliol 1493, fol. 67.

${ }^{27}$ Así lo hace el marinero y ciudadano de Barcelona Jaume Ermengol. Jaume como la mayoría de los marineros estudiados elige "meo corpori sepulturam Beate Marie de Mari in carnerio confratrum del Sant Sperit Barchinone de qua sum confrater...". Testamento otorgado en Barcelona el 13 de marzo de 1494. Antoni Benet Joan, "Primi manualis", 30 nov. 1492-4 feb. 1495 , fol. $142-142 \mathrm{v}$.
} 
servicio religioso acostumbraban a acudir a la parroquia que les correspondía.

El cura de la parroquia tenía un derechos sobre sus parroquianos, denominados derechos de parroquialidad, como por ejemplo las tasas que le debían abonar para que celebrase las bodas, bautismos y entierros, o la tasa que se pagaba a la parroquia para obtener el permiso para ser enterrado en su cementerio.

La cantidad que se pagaba por derecho de parroquialidad variaba mucho entre las gentes de mar, en los testadores estudiados oscila entre 1 y 5 sueldos.

Los testamentos analizados ${ }^{28}$ permiten apreciar que el valor de la tasa que se pagaba a la parroquia como derecho estaba relacionada con la condición social del testador y, no dependía, en general, de la importancia de la parroquia a la que pertenecía. Así, el patrón de barca Pere Caselles de Barcelona paga 5 sueldos $^{29}$ a Santa María del Mar, también paga la misma cantidad el patrón de coca Ramon Eimeric $^{30}$, mientras que el marinero Domènec Mestre, también de Barcelona, paga a Santa María del Mar 1 sueldo ${ }^{31}$.

Esta estrecha relación con la parroquia y la persistencia de la piedad en este marco religioso puede relacionarse, además, con el carácter un tanto cerrado del barrio de los pescadores y marineros en las ciudades mediterráneas $^{32}$.

Los testamentos barceloneses de los siglos XIV y XV muestran que el medio marítimo participa de la devoción general a los grandes conventos mendicantes, pero, en el caso barcelonés, los marinos que escogen la iglesia de una gran orden como lugar de sepultura, pertenecen, en buen número, a

\footnotetext{
${ }^{28}$ Pertenecientes a los notarios Bonanat Rimentol, "Manual", abril 1348-agost 1349; Berenguer Ermengol, "Manual de testamentos", 1385-1405; Bernat Nadal, "Manual", 17 abril 1389-29 octub. 1389; Pere Ullastrell, "Manual de testamentos", 1382-1385; Ramon de Massana, "Capibrevium testamentorum et codicillorum", 1371-1376: Llorenç Aragay. "Librum Testamentorum primum", 1407-1418; Guillem Jordà, "Tricesimun quartum manuale", 2 gener 1493-23 juliol 1493; Antoni Benet Joan. "Primi manualis". 30 nov. 1492-4 feb. 1495: Benet Joan. "Primum manuale", 1 marc 1501-25 féb. 1502.

${ }_{29}^{29}$ AHPB, Llorenç Aragay, "Librum testamentorum primun", (1407-1418). fol. 20-21.

${ }^{30}$ Testamento otorgado en Barcelona el 22 de junio de 1348. Bonanat Rimentol. "Manual", abril 1348-agost 1349. fol. 20v.

"AHPB, Llorenç Aragay, "Librum testamentorum primun", fol. 24v.-25

"Cfr. H. BRESC, op. cit., p. 432.
} 
los grupos profesionales más cualificados y por tanto gozan de una mejor posición económica ${ }^{33}$. Así, el patrón de coca Ramon Eimeric ${ }^{34}$ elige el cementerio de la iglesia de los agustinos de Barcelona, a pesar de ser parroquiano de Santa María del Mar, y Jaume Conesa elige el cementerio del convento de los predicadores ${ }^{35}$.

Estos últimos ejemplos no invalidan la tesis que apunta $\mathrm{H}$. Bresc para Palermo ${ }^{36} \mathrm{y}$, que se puede sostener también, para Barcelona. En esta ciudad la elección del cementerio de la iglesia de una gran orden no es tampoco una elección mayoritaria entre la gente de la mar.

La mayoría de testadores, aunque no ignoran en sus legados piadosos a los franciscanos, dominicos, agustinos y carmelitas ${ }^{37}$, selecciona, con preferencia, el cementerio de su parroquia para ser enterrado, tal como lo hace el marinero Francesc Calaf ${ }^{38}$, o el marinero Gerard Companyó ${ }^{39}$. Si bien, como bien señala Bresc, en esta elección y en la difusión de ciertos

\footnotetext{
${ }^{33}$ Aunque, también algunos marineros o esposas de marineros piden ser enterrados en el cementerio de la iglesia de alguna orden. Los casos documentados dentro de este grupo piden, con frecuencia, ser enterrados en el cementerio de los franciscanos o Fra Menors. Así consta en el testamento, ya citado, otorgado por Valença, esposa del marinero Guillem Leopardi, el 27 de diciembre de 1375, que además pide ser enterrada con el hábito de la orden y, señala que su parroquia era Sant Just. Ramon de Massana, "Capibrevium testamentorum et codicillorum", 1371-1376, fol. 52v. Aunque, Pascasia, esposa del marinero Joan Lopez, alias Medina, ciudadano de Barcelona, pide ser enterrada en el cementerio de la iglesia de los agustinos, a pesar de ser parroquiana de Santa María del Mar. Testamento otorgado el 8 de mayo de 1389. Bernat Nadal, "Manual", 17 abril 1389-29 octub. 1389.

${ }^{34}$ Bonanat Rimentol, "Manual", abril 1348-agost 1349, fol. 21.

${ }^{35}$ Además entre sus legados hace constar 5 sueldos para el citado convento y otros 5 para el de los franciscanos. Testamento otorgado el 22 de febrero de 1384. Pere Ullastrell, "Manual de testamentos". 1382-1385. fol. 7.

${ }^{36} \mathrm{H}$. BRESC, La pieté des gens de mer en Mediterranée occidentale aux dernièrs siécles du Moyen-Age, en Le genti del mare Mediterraneo a cura di Rosalba RAGOSTA, vol. prim.. Nápoles, 1981, p. 431.

${ }^{37}$ Así lo hace la ya mencionada Valença, esposa del marinero Guillem Leopardi, en su testamento datado el 27 de diciembre de 1375, al atribuir legados para la obra de la iglesia de los franciscanos y para misas en dicha iglesia. También recuerda en su última voluntad a los carmelitas y mercedarios. Ramon de Massana, "Capibrevium testamentorum et codicillorum". 1371-1376, fol. 56v.

${ }^{38}$ El marinero Francesc Calaf elige el cementerio de la iglesa de Sant Just. que era su parroquia. Testamento otorgado en Barcelona el 5 de sept. de 1371. Ramon de Massana. "Capibrevium testamentorum et codicillorum", 1371-1376, fol. 52v.

${ }^{39}$ Este marinero dispone en su testamento que quiere ser enterrado en el cementerio de su parroquia, Santa María del Mar. Testamento otorgado el 29 de abril de 1400. Berenguer Ermengol, "Manual de testaments", 1385-1405, fol. 136v.
} 
cultos tienen un papel esencial las costumbres y modas de cada zona geográfica ${ }^{40}$.

La misa era, en los últimos siglos medievales ${ }^{41}$, el sufragio por excelencia. Es posible que su importancia esté en relación, entre otras razones, con el cambio de mentalidad que se opera en esta época y con la preeminencia de la Iglesia ${ }^{42}$. Los legados para misas dependen de la voluntad del propio testador. El moribundo desea garantizarse, él mismo, el paso a la gloria, pero, aun así, depende de las plegarias de los parientes, amigos y vecinos, tiene que encontrar el sufragio en limosnas y depende de la celebración de misas, en ocasiones en cantidades sorprendentes.

Encontramos personas como el marinero Bartomeu de Trapani, natural de esta ciudad siciliana y ciudadano de Barcelona ${ }^{43}$, que dejan como heredera universal a su propia alma, es decir, los sufragios por su alma y, entre éstos, en primer lugar, las misas.

Es evidente el interés por la salvación de la propia alma a través de la Iglesia y con la colaboración de los vivos. Esta colaboración se realizará por medio de las misas de difuntos, una plegaria colectiva, un espacio de reunión entre los vivos y los muertos, una muestra de la solidaridad de los vivos que así no olvidan que pronto pueden estar entre los muertos.

Tienen, también, este sentido las disposiciones de las misas de aniversario. En esta celebración todos los parientes y amigos del difunto se encuentran de nuevo para recordarlo.

El sentido de solidaridad es, aun, más evidente en aquellos casos en los que se pide que las misas no sean sólo por el alma del testador, sino también por sus padres, cónyuge o por todos los fieles difuntos.

Por ejemplo Franscisca, viuda del marinero, Pere Carbó, pide que se celebren las treinta y tres misas de san Amador y deja además $100 \mathrm{~s}$. para misas y otras causas pías para remedio de su alma ${ }^{4+}$; o el patrón de barca Antoni Palau, ciudadano de Barcelona, que manda que se celebren noventa

\footnotetext{
${ }^{40} \mathrm{H}$. BRESC, op. cit., p. 431

${ }^{4}$ Los testamentos reiteran esta importancia de la Eucaristía como sufragio por el alma de los que abandonan este mundo.

42J. Chelini, Histoire religieuse de l'Occident Mediev'al, Paris, 1968, p. 321.

${ }^{13}$ Testamento otorgado en Barcelona el 23 de diciembre de 1410. Llorenç Aragay. "Librum testamentorum primum", 1407-1418, fol. 80 .

${ }^{H}$ Testamento otorgado el 24 de diciembre de 1407 en Barcelona. Llorenç Aragay. "Librum testamentorum primum", 1407-14182, fol. 5v-6.
} 
y nueve misas de san Amador: treinta y tres por su alma, treinta y tres por la de su primera esposa, Francesca, y otras treinta y tres por el alma de su segunda esposa, Saurina ${ }^{45}$; o el marinero Martín de Segura, ciudadano de de Barcelona pero oriundo de Castilla, que manda que "celebretur in dicta ecclesia Beate Marie de Mari et per presbiteros dicte ecclesie unum trentenarium missarum iuxta vitam sancti Amatoris", para las cuales deja treinta y tres sueldos ${ }^{46}$.

La disposición de los testadores de un ciclo de misas llamadas de san Amador es un rasgo original de Cataluña ${ }^{47}$. Se trata de un ciclo de treinta y tres misas, cuya celebración queda, como el resto de devociones y prácticas piadosas, al cuidado de los ejecutores o albaceas testamentarios. Estas misas tuvieron gran aceptación y éxito entre los testadores catalanes, son misas de carácter repetitivo, es decir, celebradas en un corto espacio de tiempo. Los testamentos del marinero Domènec Mestre $^{48}$, ciudadano de Barcelona, y del marinero Bartomeu Codina ${ }^{49}$, de la misma ciudad, son un claro reflejo de esta preferencia.

Los testamentos no detallan, sin embargo, si las misas de san Amador eran celebradas en iglesias o monasterios diferentes. Estas misas se justifican por la leyenda de san Amador y son misas que se celebran con un ritual preciso de luces, velas e invocaciones determinadas ${ }^{50}$. Para sufragar estas misas se legaban, como hemos dicho, treinta y tres sueldos, un sueldo por misa.

\footnotetext{
${ }^{45}$ Testamento otorgado en Barcelona el 18 de agosto de 1410. Llorenç Aragay, "Ídem". fol. $11-11 v-12$

${ }^{46}$ Guillem Jordà, "Tricesimum quartum manuale". 2 gener 1493-23 juliol 1493. fol. 66v.-67.

${ }^{47}$ Cfr. H. BRESC, op. cit.. p. 432. $24 \mathrm{v} .-25$.

${ }^{48}$ Testamento otorgado en Barcelona el I de marzo de 1410. Llorenç Aragay, "Ídem". fol.

${ }^{49}$ Testamento del 10 de julio de 1410. Llorenç Aragay, Íbidem, fol. 60v.-61v.

${ }^{50}$ En Cataluña estas misas seguian la siguiente organización: 7 misas dedicadas a la Virgen, en las que se encendían 7 velas; 3 misas dedicadas a la Trinidad, en las que se encendian 3 velas; 1 misa al Espíritu Santo, en la que se encendian 2 velas: 1 misa dedicada a Santa María Magdalena, en la que se encendían 2 velas; 2 misas ofrecidas a los ángeles, en las que se encendían 2 velas; 4 misas a los apóstoles, en las que se encendían 12 velas; 1 misa dedicada a los evangelistas, en la que se encendían 4 velas; I misa a la Santa Cruz, en la que se encendian 2 velas; 1 misa ofrecida a los mártires, en las que se encendían 2 velas: 1 misa dedicada a los confesores, en la que se encendian 2 velas; 1 misa ofrecida a las vírgenes, en la que se encendian 11 velas y 3 misas de difuntos, en las que se encendían 2 velas. Cfr. G. LLOMPART, Aspectes populars del purgatori medieval. en "Revista de Dialectología y tradiciones populares", XXVI (1970).
} 
Los testamentos de gentes de mar reiteran los legados para diferentes tipos de misas. Se especifica el día que se tienen que celebrar ${ }^{51}$, el número y la periodicidad y, también, el lugar e, incluso, se elige a un cura o fraile determinado para celebrarla.

Con la propia celebración de la Eucaristía se entremezclan advocaciones a santos, ofrendas de pan y luz, así como leyendas y símbolos númericos.

La presencia de varios tipos de misas obedece a la coexistencia de ideologías distintas sobre la muerte, en esta época en la que se detectan ideas tan cambiantes y contradictorias sobre la vida, la muerte, el juicio y sobre el tiempo. Puede apreciarse en numerosos testamentos que estas ideas conviven y se mezclan.

Algunos testadores disponen misas en fechas muy cercanas a la muerte para favorecer el Juicio Particular y para propiciar la separación del cuerpo y del alma; y también disponen, en ocasiones, un aniversario perpetuo, es decir, para siempre, a la espera del fin de los tiempos.

Los testamentos son poco explícitos en detalles, casi nunca se especifica si se hará o no misa de cuerpo presente. Es posible que sea una práctica bastante extendida y, que por ello, no se especifica en cada caso ${ }^{52}$.

En algún testamento, sin embargo. sí se menciona la misa de funeral cuando esta tiene alguna característica especial. Este es el caso del marinero Antoni del Barber, vecino de Barcelona, oriundo de la villa de "Beleysa comunitatis Betione", que pide "celebrari in dicta ecclesia Sancti Michaelis unam missam cantatam cum presbiteris die vero quo corpus mei sepelietur pro anima mea ..." ${ }^{\text {s3 }}$.

Hay que recordar que el rito concreto del entierro se deja, en general, en manos de los albaceas y no depende, únicamente, de la voluntad testamentaria sino de otros factores. Por ejemplo, los testamentos de algunos barceloneses muy humildes permiten observar que estos se tenían que conformar con un entierro más barato y, por lo tanto, prescindir de la misa de funeral. Tampoco los que son enterrados rápidamente después de morir reciben el sufragio de la misa, porque estas no se celebran por las tardes; ni

\footnotetext{
${ }^{51}$ El marinero Antoni del Barber pide que se celebren "missis tercii dierum capiti anni ...". Benet Joan, "Primum manuale", 1 març 1501-25 feb. 1502, fol. $7 \mathrm{v}$.

52En cambio es frecuente encontrar en los recibos de gastos de los albaceas ("rebuts de marmessoria") la mención de las misas del primer día.

${ }^{53}$ Benet Joan, "Primum manuale", 1 març 1501-25 febrer 1502, fol. 7v.
} 
los que son enterrados en fiestas litúrgicas importantes, tal como preveía el ritual.

Se celebraban, además de las ya mencionadas de san Amador ${ }^{54}$, otros tipos de misas repetitivas, las novenas, los trentenarios o misas gregorianas, todas ellas tenían lugar durante un tiempo determinado.

Estas prácticas tienen como fundamento el creer que la separación del alma y del cuerpo no se hace en un instante, sino progresivamente, eso sí, en un espacio de tiempo relativamente corto, el tercer día, el primer mes, el primer año. Es un tiempo de prueba al que es sometida el alma, un tiempo intermedio entre la vida terrenal y la eternidad.

Las restantes disposiciones piadosas que aparecen en los testamentos y libros estudiados no tienen un rasgo original: legados de cirios y velas, legados para misas, legados para obras, para pobres vergonzantes, para casar doncellas.

Siguiendo la costumbre del resto de sus contemporáneos, las gentes de mar dejan cantidades destacables e incluso la herencia universal para obras piadosas $^{55}$. La cantidad depende, evidentemente, del nivel económico, de las necesidades de la familia y de la actitud religiosa del testador y sobre todo de la influencia de la Iglesia como intermediaria ante Dios y como controladora de las relaciones entre los vivos y los muertos.

Las iglesias y los monasterios son los principales beneficiarios de los legados para causas pías, a continuación destacan los pobres -estos, a veces, no reciben el legado directamente, sino a través de una institución asistencial (hospital, cofradía o platos de los pobres) - ${ }^{56}$.

En relación con los legados piadosos, los testadores que benefician con mayor frecuencia a los pobres mendicantes y a los cautivos son los mercaderes y gente relacionada con el comercio y con el mundo del mar.

\footnotetext{
${ }^{54} \mathrm{El}$ análisis de testamentos de barceloneses de otros ámbitos socio-profesionales permiten apreciar, también, la existencia de las misas de la Trinidad y las de san Agustín. Vid. Equip Broida, El darrer comiat dels barcelonins. (Voluntats testamentàries als volts del 1400), Barcelona, 1990, libro inédito.

${ }^{55}$ Las causas pías sirven para reparar todos los pecados cometidos que no se hayan purgado en vida.

${ }^{56}$ El testamento del patrón de coca Ramon Eimeric es un claro ejemplo de la reiteración de este tipo de legados. Ramon deja a cada uno de los hospitales de pobres y casas de enfermos de Barcelona 5 sueldos; a las obras de la catedral, a las de Santa María del Mar, a las de Sant Just, a las de los conventos mercedarios, a las de los carmelitas, a las de Jonqueres, Valldonzella y, también, a la iglesia de Santa María del Pino, a cada una le hace un legado de 5 sueldos. Bonanat Rimentol, "Manual", fol. 21.
} 
Así lo hace Clara, esposa del patrón de barca Jaume Gelat, que deja 100 sueldos para su hermano Gabriel Pont que está cautivo en Argel ${ }^{57}$, el patrón de barca Martí Sánchez de Arqueta, de Castro Urdiales, que lega la cantidad necesaria para redimir a su sobrino García de Samarina, cautivo en Bugía ${ }^{58}$ o Jaume Conesa que dice en su testamento "volo dari et solvi cuidam homini marinario cuius racione ignoro, qui erat in cocha d'en Romaguera de Cauquolibero que capta fuit pro infideles sarracenos in plagia Terrachone ..."59.

Las fuentes estudiadas permiten mostrar una originalidad limitada de la piedad y la devoción de las gentes de mar. No quisiera olvidar de mencionar la importancia que tenían para estas gentes ${ }^{60}$ ciertas supersticiones $o$, si se prefiere, algunas prácticas de religiosidad popular que se alejaban del dogma oficial. Podíamos citar como factores favorecedores de tales costumbres el constante peligro que sus profesiones entrañaban, el hecho de ser consideradas gentes distintas y, también, el hecho de que sus prácticas eran mucho más difíciles de controlar en el mar que en tierra.

Por último, querría señalar que, aunque, las modas transforman la jerarquía de las veneraciones y devociones, estos aspectos remiten al mundo cultural del entorno. Las devociones y prácticas de un ámbito geográfico concreto, priman, casi siempre, sobre las veneraciones ligadas al oficio de la $\operatorname{mar}^{61}$, aunque, hay que subrayar la presencia de un espacio común mediterráneo, en el que circulan las devociones, las prácticas y los cultos.

\section{RÉSUMÉ}

Les gens de la mer du XIV et du $\mathrm{XV}^{\mathrm{c}}$ siècle constituent un groupe originel et assez fermé. Les marins avaient une image du monde avec beaucoup d'élements communs comme groupe et chacum connessaît à son tour les règles qui régissent ses collègues.

\footnotetext{
${ }^{57}$ Testamento otorgado en Barcelona en 1390. Berenguer Ermengol, "Manual de Testaments", 1385-1405, fol. 99.

${ }^{58}$ Testamento otorgado en Barcelona en 1390. Berenguer Ermengol, "Manual de Testaments", 1385-1405, fol. 30 .

${ }^{59}$ Testamento otorgado en Barcelona el 26 de febrero de 1384. Pere Ullastrell, "Manual de testaments", 382-1385, fol. 7-7v.

${ }^{(6)}$ Aun si cabe, más que para el resto de grupos sociales bajo-medievales.

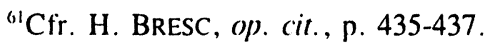


L'étude de la dévotion des gens de la mer nous permet observer que les éléments qui sónt les plus caractéristiques n'appartiennent pas au domaine ou la maîtrise de la piété. Dans cet aspect il forment un groupe perméable aux principaux courants des modes et aussi à des particularités et des moeurs de sa zone géographique de provenance.

Les dévotions et les pratiques d'un secteur géographique concret, prédominent, presque toujours, au dessus des vénérations liées au métier de la mer, bien qu'il faut souligner la présence d'un espace méditerranéen commun, parmis lequel circulent les dévotions, les pratiques et les cultes.

\section{SUMMARY}

The seamen of the XIV and XV centuries make a close, original group. Sailors, as a group, had a view of the world according to a large number of common elements, and each one knew the rules that govern their colleagues.

The study of the religious observance of these people let us know that the most typical elements have nothing to do with pity. Within the limits of this field they constitute a group which is pervious not only to the great fashions but to the peculiarities and costumes of their native geographical area.

The religious observances and methods of a specific geographical area are almost always over the cults connected to seafering though we have to take into account that there is a common Mediterranean setting where the religious practices and cults swarm about. 